\title{
Micromechanics of sheared granular layers activated by fluid pressurization
}

\author{
Hien Nho Gia Nguyen ${ }^{1}$, Luc Scholtès ${ }^{1,2}$, Yves Guglielmi ${ }^{3}$, Frédéric Victor \\ Donzé $^{4}$, Zady Ouraga ${ }^{5}$, Mountaka Souley $^{5}$ \\ ${ }^{1}$ Université de Lorraine, CNRS, GeoRessources, Nancy, France \\ ${ }^{2}$ Université Clermont Auvergne, CNRS, IRD, OPGC, Laboratoire Magmas et Volcans, Clermont-Ferrand, \\ France \\ ${ }^{3}$ Lawrence Berkeley National Laboratory, Energy Geosciences Division, Berkeley, CA, USA \\ ${ }^{4}$ Université Grenoble-Alpes, CNRS, ISTerre, Grenoble, France \\ ${ }^{5}$ Ineris, Verneuil-en-Halatte, France
}

\section{Key Points:}

- fluid induced reactivation can be either stable or unstable depending on the deformation mode

- slow steady creep is accommodated through distributed bulk deformation at subcritical stress states

- accelerated dynamic slip results from intense grain rearrangements localized within the shear band 


\begin{abstract}
Fluid pressurization of critically stressed sheared zones can trigger slip mechanisms at work in many geological processes. Using discrete element modeling, we simulate porepressure-step creep test experiments on a sheared granular layer under a sub-critical stress state to investigate the micromechanical processes at stake during fluid induced reactivation. The global response is consistent with available experiments. The progressive increase of pore pressure promotes slow steady creep at sub-critical stress states, and fast accelerated dynamic slip once the critical strength is overcome. Our multi-scale analyses show that these two emergent behaviors correlate to characteristic deformation modes: diffuse deformation during creep, and highly localized deformation during rupture. Creep corresponds to bulk deformation while rupture results from grain rotations initiating from overpressure induced unlocking of contacts located within the shear band which, consequently, acts as a roller bearing for the surrounding bulk.
\end{abstract}

\title{
Plain Language Summary
}

Fluids can be at the origin of catastrophic disasters, e.g., earthquakes related to deep subsurface fluid injections or lanslides triggered by short-term changes of hydrological conditions. It is now well assumed that these phenomena originate from mechanisms taking place in critically stressed shear zones found along tectonic faults, rock mass fractures or localized deformation bands. The increase of pore pressure promotes slip along these shear zones as confirmed by numerous experimental and numerical studies. In this work, we present computer simulations that reproduce the progressive reactivation of a granular shear zone as a result of fluid pressurization. Our simulations provide grain-scale information that improves understanding of fluid induced slip behaviors and illuminate micromechanical details of phenomenological, macroscale observations.

\section{Introduction}

Fluid overpressurization is one of the primary mechanisms at the origin of tectonic faults slip (Guglielmi et al., 2015; Cappa et al., 2019), giant landslides evolution (Cappa et al., 2014; Agliardi et al., 2020), or even glaciers deformation (Mathews, 1964; Boulton \& Hindmarsh, 1987). In all cases, slip results from mechanisms taking place within localized shear zones where most of the strain is accommodated, and it is now well assumed that these mechanisms are very sensitive to variation of pore pressure due to hydrological forcing, related to either natural or anthropogenic processes. Shear zones in tectonically active faults, at the base of large creeping landslides, or in subglacial beds, are generally sub-critically stressed (i.e., subjected to stress condition slightly lower than their instantaneous strength), and their behavior is directly related to stress related perturbations. Fluid induced reactivation can be interpreted in such a context by considering the effective stress principle combined with Mohr-Coulomb (MC) theory (King Hubbert \& Rubey, 1959). Pore fluid pressure variations modify the stress state within the shear zone by reducing the effective normal stress while the shear stress remains constant. Following MC theory and assuming that the strength of the shear zone is known (defined through its cohesion $c$ and friction coefficient $\mu$ ), the critical stress state $\tau_{c}$ at which failure will occur as a result of fluid pressurization is defined by the following criterion:

$$
\tau_{c}=c+\mu\left(\sigma_{N}-P\right)
$$

where $\sigma_{N}$ is the normal stress acting on the shear zone and $P$ is the pore fluid pressure.

Nonetheless, the MC citerion does not give any information on the mechanisms developing before or after failure. This is rather limiting since shear zones can promote different modes of slip, stable or unstable, sometimes even before the critical sress state is reached (Guglielmi et al., 2015; Scuderi \& Collettini, 2016; Scuderi et al., 2017; Cappa 
et al., 2019). The rate- and state- friction (RSF) theory offers a sound and well established framework to characterize slip behaviors (Dieterich, 1979; Ruina, 1983; Aharonov \& Scholz, 2018). However, even with modifications specifically dedicated to fluid pressure perturbations (Linker \& Dieterich, 1992), the RSF theory seems in contradiction with certain behaviors observed in nature (Cappa et al., 2019) or in laboratory (Scuderi et al., 2017), suggesting gaps in our understanding of the physical processes involved..

It is now well assumed that shear zones may accumulate displacement in two ways, through either stable creep, or unstable dynamic failure (Dieterich, 1979; Ruina, 1983). These two modes of deformation are incidentally related to the aseismic and seismic behaviors used to characterize tectonic faults slip (Reinen, 2000). As a matter of fact, loading conditions as well as micro- to large scale fault zones structure and composition strongly influence the aseismic to seismic slip transition (Bürgmann, 2018). For instance, increase in the loading rate (Mclaskey \& Yamashita, 2017), heterogeneity in the normal stress related to fault roughness (Wang \& Bilek, 2014), and width and stiffness of the fault zone may favor the transition from slow to unstable slip. At the microscale, unconsolidated clay rich granular gouges seems to favor slow slip through mechanisms of dilatant strengthening (Ikari et al., 2009), while preferential alignment of gouge minerals (foliation) and/or pressure solution processes promote velocity-strengthening and slow slip (Niemeijer \& Spiers, 2006). Seismic and aseismic slip behaviors have been both observed as a result of fluid pressurization (Cornet et al., 1998). Nevertheless, despite the compelling evidence of slip phenomena caused by pore pressure perturbations, the mechanisms at play in the transition from stable to unstable slip remain poorly understood, probably because few works have addressed the complex hydromicromechanical origins (Scuderi et al., 2017; Scuderi \& Collettini, 2018). Indeed, the response of granular gouges to stress perturbations results from frictional sliding, rolling, breaking and rearrangement of grains which are undoubtedly more intricate in the presence of hydromechanical processes.

In this paper, we propose to relate the macroscopic response of a granular shear zone to the micromechanical phenomena at work during fluid pressurization. For that matter, we developed a numerical experiment inspired by the experiments performed in (Scuderi et al., 2017) where a sub-critically stressed granular layer is subjected to a progressive pore pressure increase. The numerical experiment is built upon a numerical model based on the discrete element method (DEM) which offers a unique approach to simulate complex emergent behaviors by treating the medium under consideration as an assembly of individual particles interacting one with another according to simplified contact laws (Cundall \& Strack, 1979). DEM models have proven effective in producing emergent behaviors representative of fault gouges dynamics for several decades now (Morgan \& Boettcher, 1999; Aharonov \& Sparks, 2004; Ferdowsi et al., 2013; Ferdowsi \& Rubin, 2020). Recent efforts have actually illustrated their capability to investigate friction related fluid coupled processes (Dorostkar et al., 2017; Yang \& Juanes, 2018). In this study, we use a hydromechanically coupled DEM model to simulate fluid induced reactivation of a sub-critically stressed shear zone, so as to highlight the micromechanical processes at the origin of the emergent macroscopic slip behaviors.

\section{Method}

To investigate the hydromechanics of sheared zones, we utilized a discrete element model coupled with a pore-scale finite volume (PFV) scheme implemented in the YADE DEM software (ك̌milauer et al., 2010). The numerical medium consists of a 3D polydisperse assembly of $2.2 \mathrm{~mm} \times 2.2 \mathrm{~mm} \times 2.24 \mathrm{~mm}$ made up of 12,000 spherical particles with a uniform size distribution such that their diameters vary between $0.066 \mathrm{~mm}$ and $0.133 \mathrm{~mm}$ (the mean diameter being $D_{p}=0.1 \mathrm{~mm}$ ). The assembly is enclosed between two rigid walls in the $\mathrm{y}$ direction, and periodic boundaries are defined in both the $\mathrm{x}$ and $\mathrm{z}$ directions such that particles exit from one side and reappear on the other (Figure 1(a)). This configuration enables to simulate an infinite and homogeneous horizontal layer with a 
a)

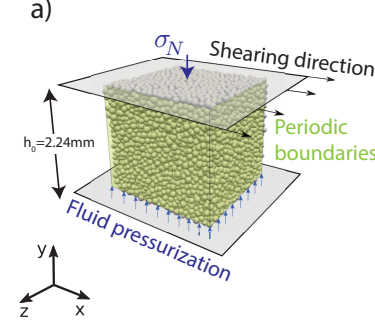

b)

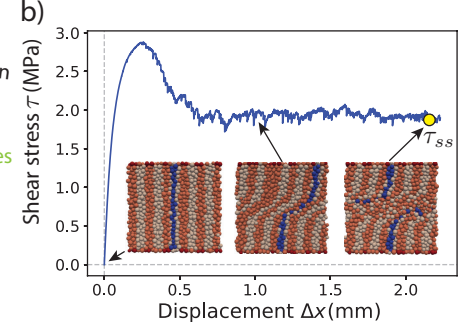

c)

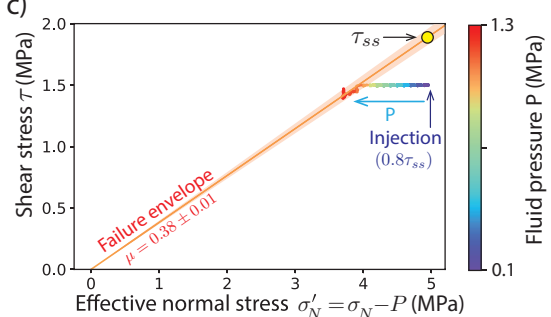

Figure 1. Numerical experiment. (a) DEM model set-up: geometry and boundary conditions defining the granular shear zone. (b) Preconditioning stage: the granular medium is sheared at constant rate under constant normal stress $\sigma_{N}$ up to a steady state defined by the steady-state strength $\tau_{s s}$ (indicated by the yellow circle on the curve). (c) Pore-pressure-step creep test: the shear stress $\tau$ is reduced and kept constant at $80 \%$ of $\tau_{s s}$ and the pore fluid pressure $P$ is then progressively increased so that the normal effective stress $\sigma_{N}^{\prime}$ decreases to reach failure.

limited computational cost without sacrificing potentially important out of plane particle reorganizations which can be of significance in terms of emerging behaviors (Hazzard \& Mair, 2003). The model's governing physics is similar to the one used by (Scholtès et al., 2015) to study the hydrodynamics of coastal sediments. Particles interact one with another through linear elastic frictional interactions. Each particle is identified by its own mass, radius and moment of inertia. An explicit solution scheme is used to integrate Newton's second law so as to update the particles' positions according to the forces they are subjected to. The method is hydromechanically coupled in the sense that deformation of the pore space caused by particles' movements induces pore pressure variations and associated interporal flow, while pore pressure changes induce hydraulic forces on the solid particles and associated stress variations. A summary of the model's formulation, the numerical parameters, as well as of the emergent properties of the simulated shear zone are provided in the supporting information.

The numerical sample is prepared by first hydrostatically compacting a cloud of randomly positioned particles up to $1 \mathrm{MPa}$ in order to generate an initially isotropic and homogeneous assembly with a controlled porosity (the porosity of the assembly is a function of the interparticle friction angle defined during the compaction phase). The sample is then subjected to a normal stress $\sigma_{N}$ of $5 \mathrm{MPa}$ in the y direction through the displacement of the top and bottom walls (the walls are frictionless during this stage). Once equilibrium is reached, the particles in contact with the walls are glued to them in order to produce a certain degree of roughness at the interfaces. The top wall is then translated at a constant velocity equal to $2 \times 10^{-5} \mathrm{~m} \mathrm{~s}^{-1}$ while the bottom wall is fixed so as to reach a steady-state strength $\tau_{s s}$ corresponding here to a cumulative shear strain $\gamma=$ $\frac{\Delta x}{h}=1$ (Figure 1(b)). The shear velocity was chosen to guarantee a quasi-static response of the system (the inertial number $I=\frac{\dot{\gamma} D_{p}}{\sqrt{\sigma_{N} / \rho_{p}}}=2.045 \times 10^{-7}$, well below the upper limit of $10^{-3}$ needed to ensure quasi-static flow as proposed in (MiDi, 2004)). This preconditioning stage enables shear to localize within the simulated medium in the form of a shear band with a thickness of approximately 10 particles, as commonly observed in granular materials (Rattez et al., 2020). The top wall is then stopped and the control mode changes from displacement-controlled to stress-controlled for the pressurization stage. Similarly to the creep experiments performed by Scuderi et al. (Scuderi et al., 2017), the shear stress $\tau$ is reduced to $80 \%$ of the steady-state strength $\tau_{s s}$ and then kept constant at this sub-critical value while the pore fluid pressure $P$ is increased step-wise to simulate a progressive reduction of the effective normal stress $\sigma_{N}^{\prime}=\sigma_{N}-P$ (Figure 1(c)). As shown in Figure 1, the servo-control is able to maintain a constant shear stress up 

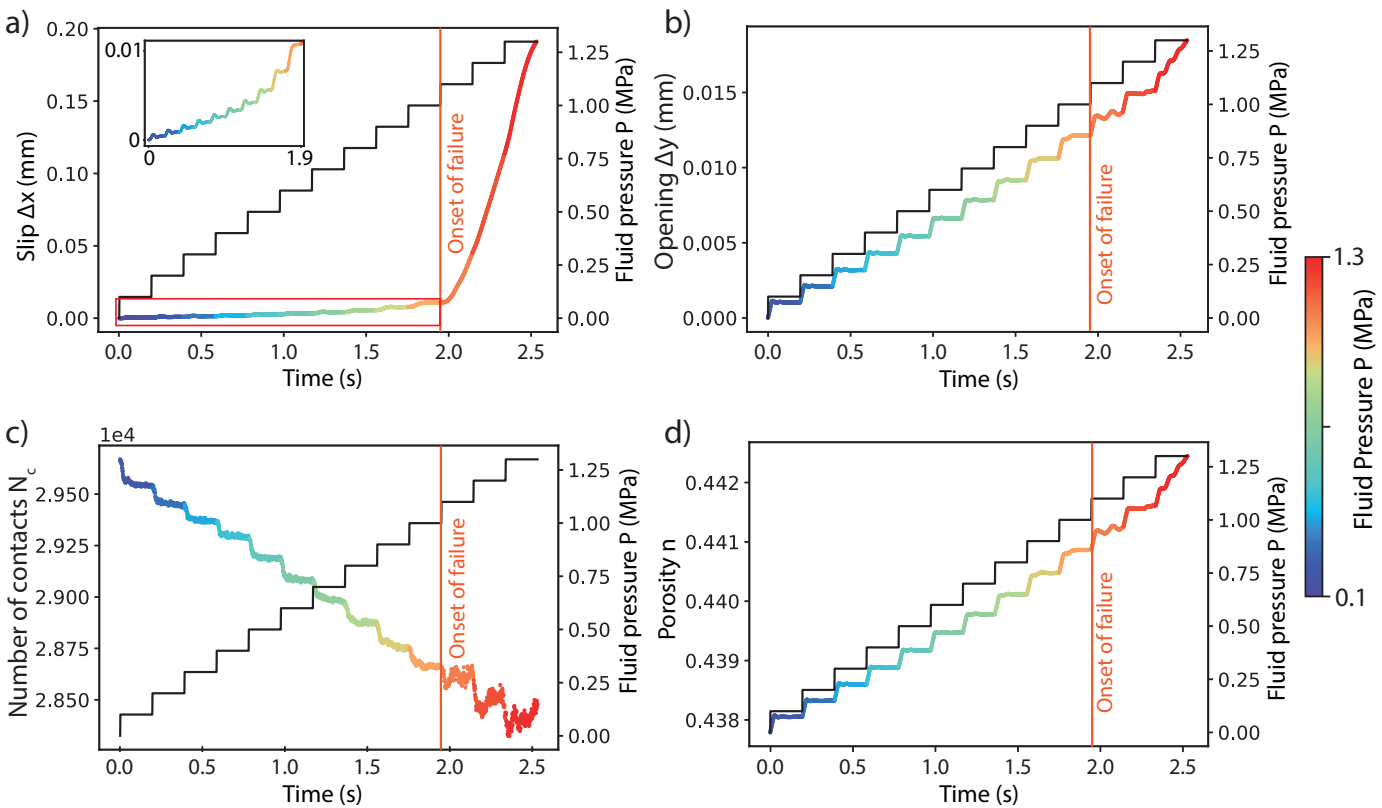

Figure 2. Response of the simulated shear zone during progressive fluid pressurization. (a) Shear displacement with a zoom in on the pre-failure stage. (b) Normal displacement. (c) Total number of sphere-sphere contacts. (d) Evolution of porosity. The curves are color-coded as functions of the fluid pressure $P$. Fluid pressure curves are plotted in black in each graph for reference.

to failure but cannot keep up afterward given the dynamic response of the medium, leading to a stress drop.

\section{Macroscopic behaviors}

The simulated emergent behavior shows strong similarities with slip behaviors observed in laboratory (Scuderi et al., 2017; Scuderi \& Collettini, 2018; Agliardi et al., 2020) and in situ (Guglielmi et al., 2015; Cappa et al., 2019) experiments under similar conditions: slow steady slip is first observed up to the critical stress state $\left(\mu \sigma_{N}^{\prime} \approx \tau_{s s}\right)$ from which accelerated slip is then triggered. Those two stages have been assimilated by Scuderi et al. (Scuderi et al., 2017) respectively to the steady-state and unstable creep behaviors observed during secondary and tertiary creeps of intact rock (Brantut et al., 2013). Unlike the laboratory experiments performed by Scuderi et al., our simulation does not show any evidence of primary creep related compaction during the preconditioning stage of the loading. This compaction is most certainly related to time-dependent processes (e.g., pressure solution) leading to grain to grain indentation that we did not take into account in our model formulation.

The first stage (secondary creep) is characterized by a quasi-linear increase of both the shear and normal displacements as functions of the fluid pressure increase. Each pressure step produces an almost instantaneous acceleration of the system before it stabilizes to a new steady state (characterized by the successive plateaux on the curves). The amplitudes of the normal displacement steps remain proportional to the pressure increase over the entire stage (Figures 2(b)) while the amplitudes of the shear displacement steps tends to slightly increase as the system approaches the critical stress state (Figures 2(a)). Similarly to what was observed in the experiments of Scuderi et al. (Scuderi et al., 2017), 
the layer suffers a systematic overall dilation resulting from the fluid pressurization. The volumetric deformation of our numerical shear zone directly correlates to its opening and is proportional to the pressure increase (Figure 2(d)). This pressure induced dilation causes a progressive loss of interparticle contacts also proportional to the pressure increase, suggesting a redistribution of some interparticle forces into hydrostatic forces (Figure 2(c)).

The second stage (tertiary creep) is characterized by a rapid acceleration of slip that spontaneously evolves into dynamic failure as suggested by the slight decrease of shear stress described by the red portion of the curve in Figure 1(c). In agreement with the experiment done by Scuderi et al. (Scuderi et al., 2017), the onset of failure corresponds almost exactly to the moment where the stress state approaches the failure envelope (defined by the Mohr-Coulomb criterion). In contradiction to what was observed in the laboratory, the medium continuously dilates at a quasi-constant rate during this accelerated slip phase with, nonetheless, a slight acceleration after significant slip has developed (visible at the last pressure step, where $P$ increases from $1.2 \mathrm{MPa}$ to $1.3 \mathrm{MPa}$ on Figure 2(b)). The lack of compaction during this accelerated slip stage is most certainly related to the fact that grain crushing is not possible in our numerical model while cataclasis and grain size reduction were systematically observed in the experiments. The oscillations in the evolution of both the opening and the number of contacts illustrate the dynamic and unstable nature of the response. The amplitudes of these oscillations are more pronounced than during the slow steady slip stage and suggest intense grains rearrangements within the medium.

We can summarize our results by saying that fluid pressurization induces slip well before reaching the critical strength. Slip remains slow and steady as long as this critical state is not reached. It accelerates and becomes dynamic afterward, with some instabilities emerging from grain scale rearrangements. Dilation persists during the entire fluid pressurization and appears to develop at a quasi-constant rate, directly proportional to the pressure increase, whatever the emergent slip behavior.

The hydromechanical behaviors predicted by our numerical shear zone shows great similarities with behaviors observed both in laboratory and in situ on shear zones presenting different natures and subjected to different stress states (Guglielmi et al., 2015; Scuderi et al., 2017; Scuderi \& Collettini, 2018; Cappa et al., 2019; Agliardi et al., 2020). Besides the relevance of the method itself and the confirmation that our DEM model constitutes a good analog to study natural shear zones, our results confirm the consistency of shear zones' response to short term pore pressure variations despite the scale effect and the added complexity of natural environments.

\section{Micromechanical processes}

In order to get further insights into the mechanisms at work at the grain scale, we discretized the shear zone into 12 equal-sized sub-layers parallel to the shearing direction to estimate the vertical distributions of the quantities presented in Figure 2. Each layer is approximately 3 particles thick. The pressurization induced variations of slip, opening, number of contacts and porosity are presented in Figure 3 as vertical profiles corresponding respectively to 2 pre-failure states and 2 post-failure states (onset of failure occurs when the fluid pressure $P$ increases from $1 \mathrm{MPa}$ to $1.1 \mathrm{MPa}$ ).

In terms of displacements (Figures 3(a,b)), shear is preferentially localized within the shear band after failure. The profiles at sub-critical states $(P=0.7$ and $0.9 \mathrm{MPa})$ show slight but limited S-bend shapes compared to the post-failure states. The S-bend shape highlights the role of the shear band on the overall response and the relative passiveness of the surrounding bulk with regards to slip. A similar but less pronounced trend can be observed for the opening displacement profiles where inflexion points can be noticed at the shear band's boundaries. As observed macroscopically in Figures 2(a,b), the open- 

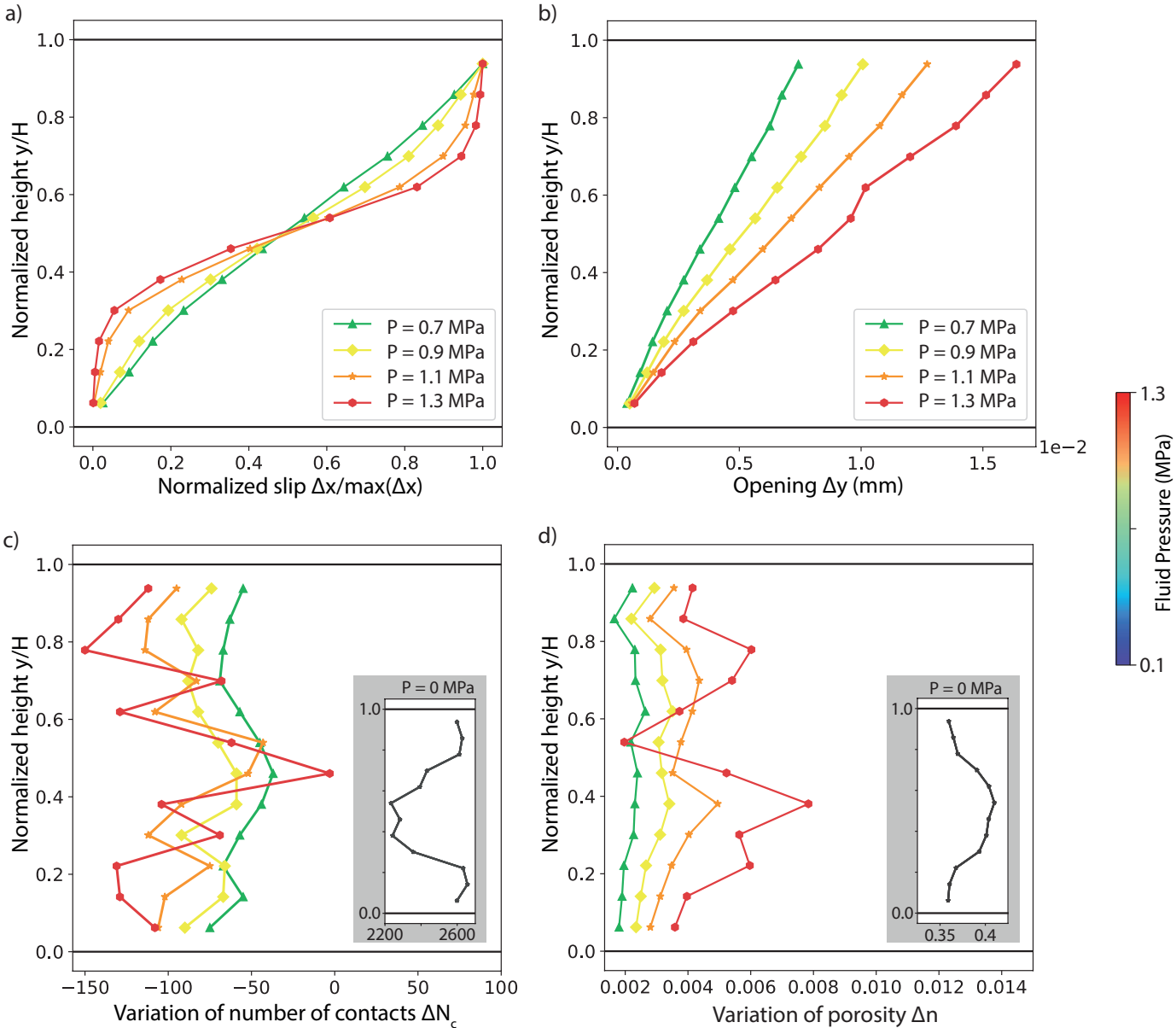

Figure 3. Profiles showing the vertical variation distributions of: (a) normalized slip, (b) opening, (c) number of contacts and (d) porosity within the shear zone at different stages of the fluid pressurization. The curves are color-coded as functions of the fluid pressure. Onset of failure occurs when the fluid pressure $P$ increases from $1 \mathrm{MPa}$ to $1.1 \mathrm{MPa}$. Inset figures in c) and d) show the vertical distributions of both the number of contacts and the porosity at the initial state, before fluid pressurization $(P=0)$. 
ing increments scale almost proportionally with the pore pressure increment at all stages, in contrast with the exponential increase of the post-failure slip increments.

The variations of number of contacts and porosity (Figures 3(cd)) clearly point out to 2 different mechanisms representative respectively of (i) the slow and steady pre-failure creep, and (ii) the accelerated and dynamic post-failure slip. Both quantities evolve quasimonotonically before failure across the entire layer, indicating an homogeneous response of the bulk to the pressurization: every increment of pore pressure leads to a loss of contacts as well as to a porosity increase which, as suggested by the macroscopic responses (Figures 2(cd)), are quasi-proportional to the pressure increase. After failure $(P>1.1 \mathrm{MPa})$, both quantities highlight a localization of the deformation inside the shear band where contacts are gained and porosity decreases as described by the clear midheight spike observed at $P=1.3 \mathrm{MPa}$ in both profiles. This indicates shear induced compaction within the shear band. Interestingly, the evolutions of both quantities outside the shear band follow the trends observed prior to failure, namely, loss of contacts and porosity increase. The coexistence of these somewhat opposite volumetric trends in and out of the shear band suggests deformation mechanisms taking place at the interfaces between the bulk and the shear band where a large amount of the opening is accommodated as suggested by the 2 spikes in the porosity profile at $P=1.3 \mathrm{MPa}\left(\frac{y}{H} \approx 0.4,0.8\right)$, in conjunction with a noticeable gain of contacts below these interfaces $\left(\frac{y}{H} \approx 0.3,0.7\right)$. These post-failure profiles suggest sharply defined sub-horizontal structures that could actually be assimilated to the so-called Y-shears commonly observed in sheared materials in natural context (Reinen, 2000) and in experiments, either in the laboratory (Scuderi \& Collettini, 2018) or in numerical analogs (Morgan \& Boettcher, 1999).

Our observations suggest a high degree of particle rearrangement once accelerated slip occurs, leading to the generation of a hyperactive layer (the shear band) effectively disconnected from the surrounding material by discrete structures. By tracking the rotation of particles during the reactivation process (Figure 4(a)), we confirm the micromechanisms at play: slow steady creep corresponds to bulk deformation due to interlocked particles, while accelerated dynamic slip is mainly accommodated by interparticle rolling concentrated within the shear band. As discussed by Morgan and Boettcher (Morgan \& Boettcher, 1999), shear loads within granular materials are borne by chains of particles carrying high forces. These chains evolve during shear and eventually fail due to interparticle rolling. We posit that post-failure accelerated slip is triggered by the sudden collapse of these force chains (a mechanisms identified as force-chain buckling in the literature by, e.g., (Tordesillas, 2007)) within the shear band. As shown in Figure 4(b), the interparticle contact forces align to the direction of the maximum principal stress for both pre-failure and post-failure states (inclined at approximately $45^{\circ}$ to the loading direction). Strong force chains are predominantly located within the shear band before failure. The same strong force chains actually persist up to failure (Figure 4(b)) and disappear once failure occurs. Once particles start to roll, the entire shear band acts as a roller bearing which promotes slip of the surrounding bulk, similarly to what can be observed when the medium is sheared under steady state conditions (see supporting information, Figure S3). One can note that the slip rate tends to stabilize once this roller bearing is activated. Strong interparticle forces still concentrate within the shear band as a result of its compaction, but in a more diffuse manner. Interestingly, the distribution of sliding contacts (Figure 4(c)) does not directly correlate with particle rotations, specially just before the onset of failure $(P=1 \mathrm{MPa})$ where particles starts to slide intensively inside the shear band, as opposed to what can be observed at lower pore pressures $(e . g ., P=0.7 \mathrm{MPa}$ ) where interparticle slips are scarcely distributed over the entire system. The interparticle slips occurring inside the shear band just before the onset of failure are clear precursors of the force chain collapses that lead to particle rolling and overall failure. Post-failure, interparticle slips mainly occur inside the shear band where most particle rearrangements are concentrated, but some particles also slip outside of the shear band. This is slightly different from what can be observed when the medium 
is sheared at constant rate where interparticle slips are concentrated within the shear band (see supporting information, Figure S3), most probably due to the dynamic character of the stress-controlled response versus the quasi-static strain-controlled response.

To sum up, pre-failure slow steady creep is associated with diffuse bulk deformation while post-failure accelerated dynamic slip results from localized deformation processes taking place within the shear band. Similarly to what was observed in laboratory experiments on a saturated granular till (Rathbun \& Marone, 2010), the pressurization of the layer induces a progressive transition from distributed shear to localized deformation. Evidence of this bi-modal accommodation of slip has also been observed in dry serpentinite gouge by Reinen (Reinen, 2000) who concluded that the microstructures that form during shear may be used to identify the seismic behavior of natural fault zones. Our numerical results tend to confirm her conclusions stating that stable fault creep results from distributed deformation, while localized deformation favors unstable dynamic slip. Before failure, grain reorganizations are very limited and the overall response of the shear zone is driven by bulk deformation. Once failure occurs, the shear band concentrates all the grain rearrangements at the origin of the accelerated slip. As suggested by Morgan and Boettcher (Morgan \& Boettcher, 1999), failure is directly correlated to grain rotations which promote a dynamic response of the system.

\section{Concluding remarks}

We used a discrete element model to analyze the micromechanics of fluid induced reactivation of a sub-critically stressed granular shear zone. We were able to reproduce typical slip behaviors observed both in laboratory and in situ experiments: at constant shear stress, fluid pressurization reduces the normal stress and promotes (i) slow steady slip before the critical strength is reached, and (ii) accelerated dynamic slip afterward. Our micromechanical analysis show that these two slip behaviors are respectively related to two distinct deformation modes: (i) a distributed mode where the entire layer deforms homogeneously in response to the pressure increase, and (ii) a localized mode where the shear band acts as a roller bearing for the surrounding bulk. The transition between these two deformation modes results from the rupture of interparticle force chains located within the shear band. By reducing the effective stress, fluid overpressurization promotes interparticle slips along these force chains which eventually lead to intense particle rearrangements once the critical strength is reached. Pre-failure slip is mainly accommodated by dilation induced elastic shear distributed over the entire bulk, while post-failure slip is mainly accommodated through particle rolling concentrated within the shear band. Our results suggest that grain rotations promote slip instabilities during fluid pressurization of granular shear zones.

Our numerical experiment constitutes a simplification of realistic configurations and it is evident that the simplicity of such an idealized system affect its mechanical behavior. For instance, the shape of particles and their size distribution can both influence the local deformation mechanisms as well as the overall volumetric behavior of the simulated material (Morgan \& Boettcher, 1999; Mair et al., 2002). Nonetheless, several studies have shown that discrete element models can be considered as a good first approximation to understand the complexity of the mechanisms that characterize shear zones during deformation (Morgan \& Boettcher, 1999; Aharonov \& Sparks, 2004; Mair \& Hazzard, 2007; Rathbun et al., 2013). Even though the present study provides insight into the micromechanisms at work in pressurized granular shear zones, additional work is needed to fully characterize fluid induced slip behaviors in natural shear zones. For instance, beside the influence of key loading parameters such as the injection rate or the normal stress that needs to be investigated, the introduction of time-dependent physics in the model formulation as suggested for example by (Van Den Ende et al., 2018) would allow to discuss fluid induced slip behaviors in the context of the rate- and state- friction theory, while the consideration of crushable grains as proposed for example by (Abe \& Mair, 2009) 

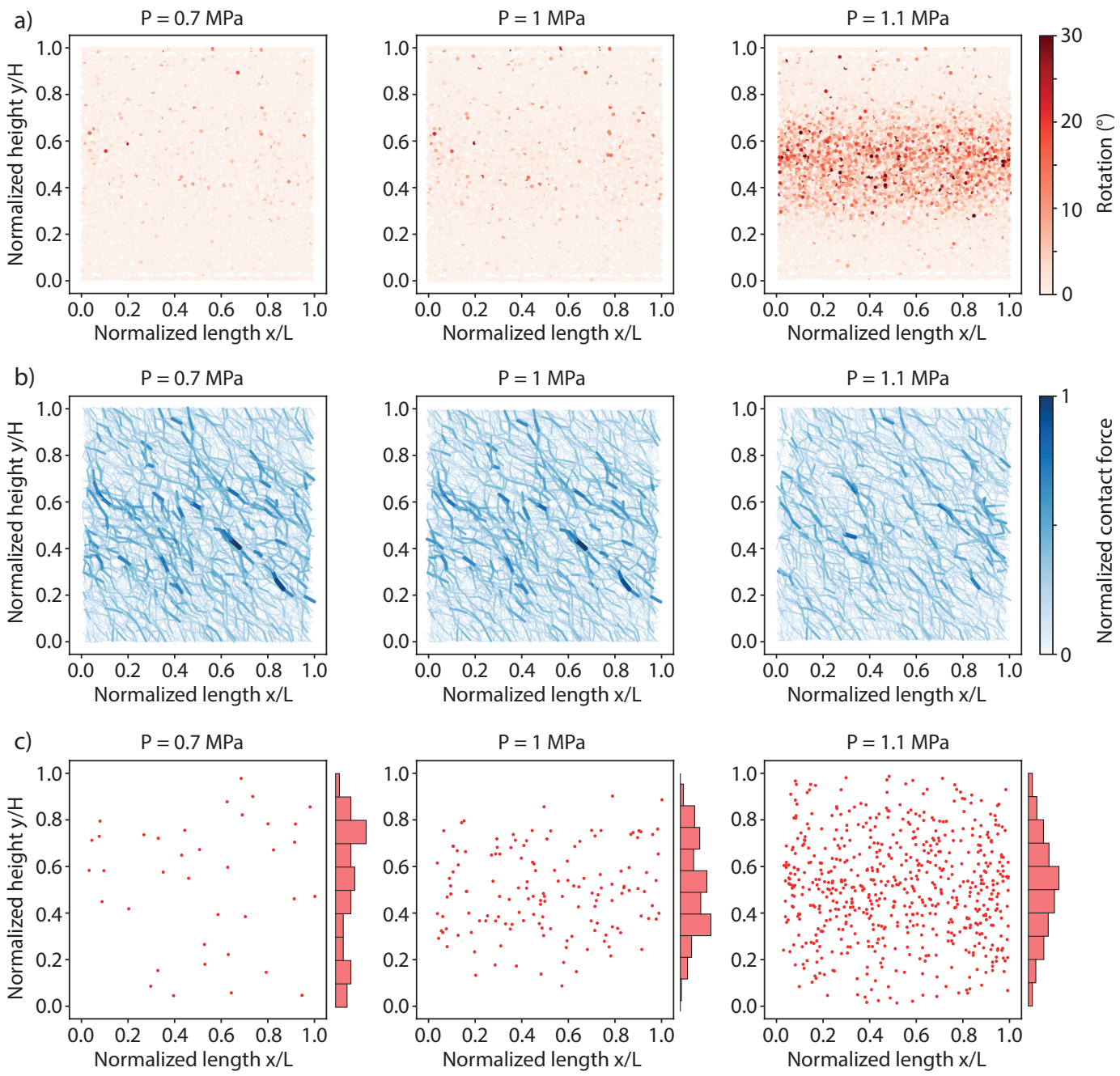

Figure 4. Microscale analyses on a vertical slice extracted from the sample at different stages of the pressurization: $P=0.7 \mathrm{MPa}, P=1 \mathrm{MPa}$, and $P=1.1 \mathrm{MPa}$. (a) Spatial distribution maps of accumulated particle rotations. (b) Interparticle contact force network (the color intensity and thicknesses of the line segments are proportional to the contact force magnitude, the contact forces are normalized similarly for all considered stages). (c) Spatial distribution of sliding contacts with their density histograms. Particle-wall contacts are omitted to focus on what happens within the sample. Onset of failure occurs when the fluid pressure $P$ was increased from $1 \mathrm{MPa}$ to $1.1 \mathrm{MPa}$. 
would give further insights into the deformation process since grain fracturing plays a key role in the evolution of granular gouges during shear.

\section{Acknowledgments}

This work was supported partly by the french PIA project "Lorraine Université d'Excellence", reference ANR-15-IDEX-04-LUE. The data related to this paper can be accessed via an online repository from the link https://datadryad.org/stash/share/

x1FncqSWkSUCzWenWqXhLra53sZXFHBC9o6qv0oRhYE, or by contacting the corresponding authors. The software used for this study is open source and publicly available as cited in the main text.

\section{References}

Abe, S., \& Mair, K. (2009). Effects of gouge fragment shape on fault friction: New 3d modelling results. Geophysical Research Letters, 36(23). doi: 10.1029/ 2009GL040684

Agliardi, F., Scuderi, M. M., Fusi, N., \& Collettini, C. $\quad$ (2020). Slow-to-fast transition of giant creeping rockslides modulated by undrained loading in basal shear zones. Nature Communications, 11(1), 1352. doi: 10.1038/ s41467-020-15093-3

Aharonov, E., \& Scholz, C. H. (2018). A physics-based rock friction constitutive law: Steady state friction. Journal of Geophysical Research: Solid Earth, 123(2), 1591-1614. doi: 10.1002/2016JB013829

Aharonov, E., \& Sparks, D. (2004). Stick-slip motion in simulated granular layers. Journal of Geophysical Research: Solid Earth, 109(B9). doi: 10.1029/ 2003JB002597

Boulton, G. S., \& Hindmarsh, R. C. A. (1987). Sediment deformation beneath glaciers: Rheology and geological consequences. Journal of Geophysical Research: Solid Earth, 92(B9), 9059-9082. doi: 10.1029/JB092iB09p09059

Brantut, N., Heap, M. J., Meredith, P. G., \& Baud, P. (2013). Time-dependent cracking and brittle creep in crustal rocks: A review. Journal of Structural Geology, 52, 17-43. doi: 10.1016/j.jsg.2013.03.007

Bürgmann, R. (2018, August). The geophysics, geology and mechanics of slow fault slip. Earth and Planetary Science Letters, 495, 112-134. Retrieved 2021-05-10, from https://www.sciencedirect.com/science/article/pii/ S0012821X18302760 doi: 10.1016/j.epsl.2018.04.062

Cappa, F., Guglielmi, Y., Viseur, S., \& Garambois, S. (2014). Deep fluids can facilitate rupture of slow-moving giant landslides as a result of stress transfer and frictional weakening. Geophysical Research Letters, 41(1), 61-66. doi: 10.1002/2013GL058566

Cappa, F., Scuderi, M. M., Collettini, C., Guglielmi, Y., \& Avouac, J.-P. (2019). Stabilization of fault slip by fluid injection in the laboratory and in situ. Science Advances, 5(3), eaau4065.

Cornet, F. H., Helm, J., Poitrenaud, H., \& Etchecopar, A. (1998). Seismic and aseismic slips induced by large-scale fluid injections. In S. Talebi (Ed.), Seismicity associated with mines, reservoirs and fluid injections (p. 563-583). Basel: Birkhäuser Basel.

Cundall, P. A., \& Strack, O. D. L. (1979). A discrete numerical model for granular assemblies. Géotechnique, 29(1), 47-65. doi: 10.1680/geot.1979.29.1.47

Dieterich, J. H. (1979). Modeling of rock friction: 1. experimental results and constitutive equations. Journal of Geophysical Research: Solid Earth, 84(B5), 21612168. doi: 10.1029/JB084iB05p02161

Dorostkar, O., Guyer, R. A., Johnson, P. A., Marone, C., \& Carmeliet, J. (2017). On the micromechanics of slip events in sheared, fluid-saturated fault gouge. 
Geophysical Research Letters, 44(12), 6101-6108. doi: 10.1002/2017GL073768

Ferdowsi, B., Griffa, M., Guyer, R. A., Johnson, P. A., Marone, C., \& Carmeliet, J. (2013). Microslips as precursors of large slip events in the stick-slip dynamics of sheared granular layers: A discrete element model analysis. Geophysical Research Letters, 40(16), 4194-4198. doi: 10.1002/grl.50813

Ferdowsi, B., \& Rubin, A. M. (2020). A granular physics-based view of fault friction experiments. Journal of Geophysical Research: Solid Earth, 125(6), e2019JB019016. doi: 10.1029/2019JB019016

Guglielmi, Y., Cappa, F., Avouac, J.-P., Henry, P., \& Elsworth, D. (2015). Seismicity triggered by fluid injection-induced aseismic slip. Science, 348(6240), 12241226. doi: $10.1126 /$ science.aab0476

Hazzard, J. F., \& Mair, K. (2003). The importance of the third dimension in granular shear. Geophysical Research Letters, 30(13). doi: 10.1029/2003GL017534

Ikari, M. J., Saffer, D. M., \& Marone, C. (2009). Frictional and hydrologic properties of clay-rich fault gouge. Journal of Geophysical Research: Solid Earth, 114(B5). Retrieved 2021-05-10, from https://agupubs .onlinelibrary.wiley.com/doi/abs/10.1029/2008JB006089 (_eprint: https://agupubs.onlinelibrary.wiley.com/doi/pdf/10.1029/2008JB006089) doi: https://doi.org/10.1029/2008JB006089

King Hubbert, M., \& Rubey, W. W. (1959). Role of fluid pressure in mechanics of overthrust faulting: I. mechanics of fluid-filled porous solids and its application to overthrust faulting. Geological Society of America Bulletin, 70(2), 115-166.

Linker, M. F., \& Dieterich, J. H. (1992). Effects of variable normal stress on rock friction: Observations and constitutive equations. Journal of Geophysical Research: Solid Earth, 97(B4), 4923-4940. doi: 10.1029/92JB00017

Mair, K., Frye, K. M., \& Marone, C. (2002). Influence of grain characteristics on the friction of granular shear zones. Journal of Geophysical Research: Solid Earth, 107 (B10), ECV 4-1-ECV 4-9. Retrieved from https:// agupubs.onlinelibrary.wiley.com/doi/abs/10.1029/2001JB000516 doi: https://doi.org/10.1029/2001JB000516

Mair, K., \& Hazzard, J. F. (2007). Nature of stress accommodation in sheared granular material: Insights from 3d numerical modeling. Earth and Planetary Science Letters, 259(3), 469-485. doi: 10.1016/j.epsl.2007.05.006

Mathews, W. H. (1964). Water pressure under a glacier. Journal of Glaciology, 5(38), 235-240. doi: 10.3189/S0022143000028811

Mclaskey, G. C., \& Yamashita, F. (2017). Slow and fast ruptures on a laboratory fault controlled by loading characteristics. Journal of Geophysical Research: Solid Earth, 122(5), 3719-3738. Retrieved 2021-05-10, from https://agupubs .onlinelibrary.wiley.com/doi/abs/10.1002/2016JB013681 (_eprint: https://agupubs.onlinelibrary.wiley.com/doi/pdf/10.1002/2016JB013681) doi: https://doi.org/10.1002/2016JB013681

MiDi, G. (2004). On dense granular flows. The European Physical Journal E, 14, 341-365. doi: 10.1140/epje/i2003-10153-0

Morgan, J. K., \& Boettcher, M. S. (1999). Numerical simulations of granular shear zones using the distinct element method: 1. shear zone kinematics and the micromechanics of localization. Journal of Geophysical Research: Solid Earth, 104(B2), 2703-2719. doi: 10.1029/1998JB900056

Niemeijer, A. R., \& Spiers, C. J. (2006, December). Velocity dependence of strength and healing behaviour in simulated phyllosilicate-bearing fault gouge. Tectonophysics, 427(1), 231-253. Retrieved 2021-05-10, from https:// www.sciencedirect.com/science/article/pii/S0040195106003878 doi: 10.1016/j.tecto.2006.03.048

Rathbun, A. P., \& Marone, C. (2010). Effect of strain localization on frictional behavior of sheared granular materials. Journal of Geophysical Research: Solid Earth, 115(B1). doi: 10.1029/2009JB006466 
Rathbun, A. P., Renard, F., \& Abe, S. (2013). Numerical investigation of the interplay between wall geometry and friction in granular fault gouge. Journal of Geophysical Research: Solid Earth, 118(3), 878-896. doi: 10.1002/jgrb.50106

Rattez, H., Shi, Y., Sac-Morane, A., Klaeyle, T., Mielniczuk, B., \& Veveakis, M. (2020). Effect of grain size distribution on the shear band thickness evolution in sand. Géotechnique, 0(0), 1-39. doi: 10.1680/jgeot.20.P.120

Reinen, L. A. (2000). Seismic and aseismic slip indicators in serpentinite gouge. Geology, 28 (2), 135-138. doi: 10.1130/0091-7613(2000)28<135:SAASII $>2.0$.CO; 2

Ruina, A. (1983). Slip instability and state variable friction laws. Journal of Geophysical Research: Solid Earth, 88(B12), 10359-10370. doi: 10.1029/JB088iB12p10359

Scholtès, L., Chareyre, B., Michallet, H., Catalano, E., \& Marzougui, D. Modeling wave-induced pore pressure and effective stress in a granular seabed. Continuum Mechanics and Thermodynamics, 27(1), 305-323. doi: 10.1007/s00161-014-0377-2

Scuderi, M., \& Collettini, C. (2016). The role of fluid pressure in induced vs. triggered seismicity: insights from rock deformation experiments on carbonates. Scientific Reports, 6(24852). doi: 10.1038/srep24852

Scuderi, M., \& Collettini, C. (2018). Fluid Injection and the Mechanics of Frictional Stability of Shale-Bearing Faults. Journal of Geophysical Research: Solid Earth, 123(10), 8364-8384. doi: 10.1029/2018JB016084

Scuderi, M., Collettini, C., \& Marone, C. (2017, November). Frictional stability and earthquake triggering during fluid pressure stimulation of an experimental fault. $\quad$ Earth and Planetary Science Letters, 477, 84-96. $\quad$ doi: 10.1016/j.epsl.2017.08.009

Tordesillas, A. (2007). Force chain buckling, unjamming transitions and shear banding in dense granular assemblies. Philosophical Magazine, 87(32), 4987-5016. doi: 10.1080/14786430701594848

Van Den Ende, M. A., Marketos, G., Niemeijer, A., \& Spiers, C. (2018). Investigating compaction by intergranular pressure solution using the discrete element method. Journal of Geophysical Research: Solid Earth, 123(1), 107-124. doi: 10.1002/2017JB014440

Wang, K., \& Bilek, S. L. (2014, January). Invited review paper: Fault creep caused by subduction of rough seafloor relief. Tectonophysics, 610, 1-24. Retrieved 2021-05-10, from https://www. sciencedirect.com/science/article/pii/ S0040195113006896 doi: 10.1016/j.tecto.2013.11.024

Yang, Z., \& Juanes, R. (2018, Feb). Two sides of a fault: Grain-scale analysis of pore pressure control on fault slip. Physical Review E, 97, 022906. doi: 10 .1103/PhysRevE.97.022906

Šmilauer, V., Catalano, E., Chareyre, B., Dorofeenko, S., Duriez, J., Gladky, A., ... others (2010). Yade reference documentation. Yade Documentation, 474(1). 